\title{
CARACTERIZAÇÃO DO SUCO DE AMORA-PRETA ELABORADO EM EXTRATOR CASEIRO'
}

\author{
Renata Vieira da MOTA ${ }^{2, *}$
}

\begin{abstract}
RESUMO
O trabalho objetivou avaliar a composição físico-química, aceitabilidade e efeito do armazenamento na qualidade do suco de amorapreta elaborado em um extrator caseiro. Os frutos foram avaliados quanto à composição físico-química e separados de acordo com o teor de antocianinas em três grupos: acima de $160 \mathrm{mg} / 100 \mathrm{~g}$ (Guarani), de 120-160 mg/100 g (Caingangue e Comanche) e abaixo de $120 \mathrm{mg} / 100 \mathrm{~g}$ (Tupy, Seleção 97 e Cherokee). Os sucos foram pasteurizados e analisados quanto aos teores de pH, sólidos solúveis totais, acidez total titulável, carboidratos solúveis, extrato seco e antocianinas. A extração por $2 \mathrm{~h}$ apresentou rendimento de $84 \%$. As antocianinas foram os compostos que sofreram alteração mais significativa na elaboração do suco, com redução média de $42 \%$. O suco apresentou aceitabilidade superior a 85\%. O armazenamento em adega ou sob refrigeração alterou de forma significativa apenas o teor de antocianinas dos sucos, cuja degradação foi menor nas amostras conservadas sob refrigeração.

Palavras-chave: Rubus sp., qualidade, antocianinas, armazenamento, aceitabilidade.
\end{abstract}

\section{SUMMARY}

CHARACTERISATION OF BLACK-BERRY JUICE PREPARED IN A DOMESTIC EXTRACTOR. The aim of this study was the evaluation of physico-chemical composition, acceptability and storage effects on the quality of black-berry juice prepared in a domestic extractor. Fruits were analysed for their chemical properties and divided in three groups, according to anthocyanin content: above $160 \mathrm{mg} / 100 \mathrm{~g}$ (Guarani), 120-160 mg/100 g (Caingangue and Comanche) and below $120 \mathrm{mg} / 100 \mathrm{~g}$ (Tupy, Seleção 97, Cherokee). The pasteurised juices were analysed for parameters related to quality, such as $\mathrm{pH}$, total soluble sugars, titratable acidity, dry extract and anthocyanin content. Extraction for two hours resulted in a yield of $84 \%$. Juice extraction reduced the anthocyanin content in average $42 \%$. The acceptability was over $85 \%$. Storage in wine cellar or refrigerator reduced the anthocyanin content of the juices, whose degradation was smaller in samples stored under refrigeration.

Keywords: Rubus sp., quality, anthocyanins, storage, acceptability.

\section{1 - INTRODUÇÃO}

As pequenas frutas vêm despertando a atenção dos produtores e dos consumidores, principalmente após as notícias divulgadas na mídia a respeito dos benefícios que proporcionam ao organismo, devido à presença de elevado teor de compostos fenólicos com poder antioxidante $[7,8]$. Do grupo das pequenas frutas que abrange as culturas de morango, framboesa, mirtilo e amora-preta, o mirtilo é classificado como a fruta mais rica em antocianinas, com teores que variam de 93 a $280 \mathrm{mg}$ cianidina/100 g peso fresco conforme a cultivar [3], seguido pela framboesapreta (até 197,2 mg/100 g), amora-preta (171,6 mg/100 g) e morango ( $40 \mathrm{mg} / 100 \mathrm{~g}$ ) [23].

As antocianinas, compostos classificados como flavonóides, são pigmentos solúveis em água que conferem as várias nuanças entre laranja, vermelho e azul, exibidas pelas frutas, hortaliças, folhas, flores e raízes. Ultimamente, relatos científicos têm demonstrado que as antocianinas apresentam efeitos fisiológicos capazes de reduzir o risco de doenças [14].

\footnotetext{
${ }^{1}$ Recebido para publicação em 24/1/2005. Aceito para publicação em 28/4/2006 (001467)

${ }^{2}$ Estação Experimental de Caldas (Epamig)

Avenida Santa Cruz, 500, Caldas (MG)

Caixa Postal 33, CEP 37780-000.

E-mail: renata@epamigcaldas.gov.br

"A quem a correspondência deve ser enviada
}

KUSKOSKI et al. [12] determinaram a atividade antioxidante de pigmentos antociânicos e obtiveram atividade semelhante ou superior à do Trolox, um antioxidante sintético padrão. Dos cinco pigmentos antociânicos estudados, delfinidina e cianidina 3-glicosídeo apresentaram atividade duas vezes superior ao Trolox, indicando que estes compostos possuem potente propriedade antioxidante. As espécies do gênero Rubus são descritas como fontes naturais de antocianinas, glicosídeos de cianidina e em alguns casos, glicosídeos de pelargonidina, com maior concentração dos pigmentos nos frutos [17].

HASSIMOTTO et al. [10] identificaram os compostos fenólicos de cinco cultivares de amora-preta e em todos os casos, a cianidina foi o pigmento predominante contribuindo com aproximadamente $66-80 \%$ do total de antocianinas.

No Brasil, a cultura da amora-preta foi introduzida pela Estação Experimental de Pelotas, atual Embrapa Clima Temperado, no Rio Grande do Sul, na década de 70, e desde então seu cultivo vem crescendo nos Estados do Rio Grande do Sul, São Paulo e Minas Gerais, com a introdução e adaptação de novas cultivares [1].

Entretanto, devido à estrutura frágil e alta atividade respiratória dos frutos, sua vida pós-colheita é relativamente curta, sendo os frutos comercializados preferencialmente na forma industrializada. Os frutos podem ser congelados, enlatados, processados na forma de polpa para utilização em produtos lácteos (como matéria-prima ou aditivo de cor e sabor), sucos e geléias [1,2]. 
Devido ao aumento no interesse pelo cultivo da amoreira-preta e escassez de trabalhos quanto ao aproveitamento industrial dos frutos, objetivou-se avaliar o efeito do processamento e do armazenamento na qualidade do suco de amora-preta elaborado a partir de técnicas acessíveis aos pequenos produtores.

\section{2 - MATERIAL E MÉTODOS}

Foram utilizados frutos das cultivares Tupy, Guarani, Caingangue, Comanche, Cherokee e Seleção 97, obtidos no banco de germoplasma da Estação Experimental de Caldas (Epamig/FECD), safra 2003/2004.

Logo após a colheita, os frutos foram lavados em água clorada ( $25 \mathrm{mg} . \mathrm{L}^{-1}$ cloro livre) e selecionados quanto à sanidade. Após drenagem do excesso de água, foram acondicionados em bandejas e mantidos em freezer até o congelamento. Os frutos congelados foram colocados em embalagens plásticas transparentes com fecho hermético e mantidos em freezer doméstico a $-18^{\circ} \mathrm{C}$ até o uso.

Antes da elaboração do suco, as cultivares foram avaliadas quanto às características físico-químicas dos frutos. Para evitar o efeito da época de colheita (início, meio, final de safra) na avaliação das cultivares, uma unidade amostral consistiu de um número igual de frutos obtidos de cada colheita. Os frutos foram mantidos à temperatura ambiente até o descongelamento. Em seguida, foram triturados em liquidificador por um minuto e peneirados para obtenção da polpa.

A polpa das diferentes cultivares foi avaliada quanto ao $\mathrm{pH}$, sólidos solúveis totais (SST), acidez total titulável (expressa como porcentagem de ácido cítrico), umidade [11], carboidratos [5] e antocianinas totais [ 15, 18, 19]. A extração das antocianinas totais foi feita em metanol acidificado com HCL $1 \%$ e o conteúdo estimado como cianidina-3-glicosídeo, usando um coeficiente de absortividade molar de 26.900 L.cm ${ }^{-1} \cdot \mathrm{mg}^{-1}$ e peso molecular de 449,2 g/mol.

O espectro de absorção das antocianinas situa-se na faixa de $500 \mathrm{~nm}$, tendo sido detectada a $510 \mathrm{~nm}$ em polpa de morango [4], $520 \mathrm{~nm}$ [17] e $532 \mathrm{~nm}$ [18, 20] em amora e $535 \mathrm{~nm}$ em groselha [13]. Neste trabalho, o teor de antocianinas foi determinado em uma faixa de absorção de 480 a $580 \mathrm{~nm}$ a fim de determinar o comprimento de onda mais adequado para as cultivares avaliadas (Figura 1).

O comprimento de onda de $535 \mathrm{~nm}$ proporcionou o pico máximo de absorbância para as antocianinas totais extraídas de todas as cultivares. Segundo ROMMEL, WROLSTAD e HEATHERBELL [22] e HASSIMOTTO et al. [10], a principal forma de antocianina encontrada em frutos de amora-preta corresponde à forma cianidina3-glicosídeo, cujo pico de absorção situa-se na faixa de $530 \mathrm{~nm}$ [3]. Desta forma, estabeleceu-se o comprimento de onda de $532 \mathrm{~nm}$ para a determinação do teor de antocianinas totais como indicado por PERKINS-VEAZIE et al. [18] e PERKINS-VEAZIE, COLLINS e CLARK [20].

O perfil espectral (Figura 1) indicou a separação das cultivares em três grupos de acordo com o teor de anto-

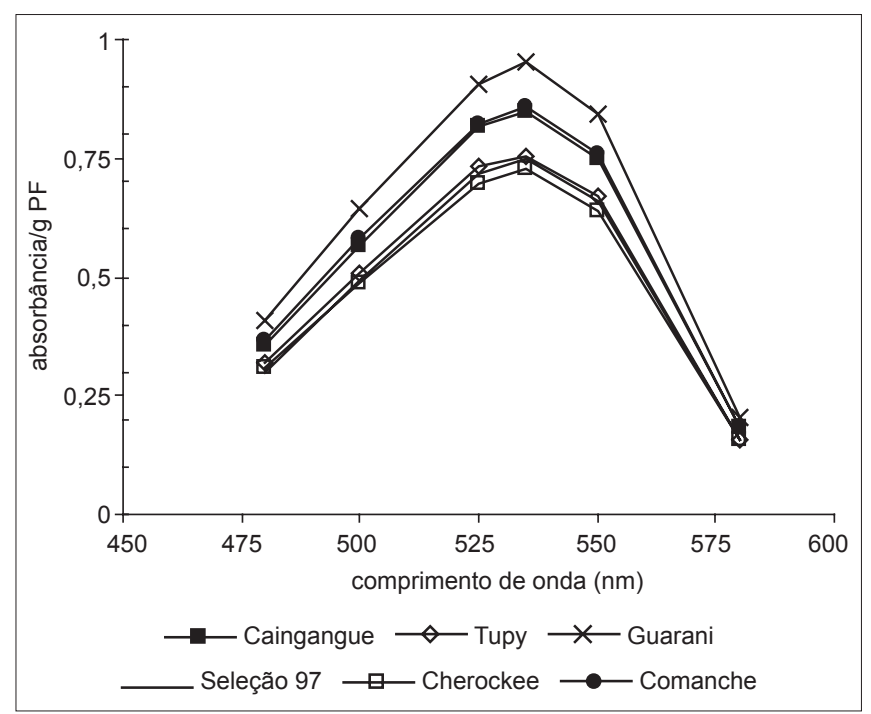

FIGURA 1 - Espectro de absorção das antocianinas totais do extrato de polpa de seis cultivares de amora-preta, obtidas no banco de germoplasma da Estação Experimental de Caldas (Epamig/FECD), safra 2003/2004

cianinas totais. Desta forma, para a elaboração do suco, os frutos foram agrupados em grupo A (teor superior a $160 \mathrm{mg}$ cianidina/100 g) representado pela cultivar Guarani; grupo B (entre 120 e $160 \mathrm{mg}$ cianidina/100 g) composto pelas cultivares Caingangue e Comanche e grupo C (teor inferior a $120 \mathrm{mg}$ cianidina/100 g) formado pelas cultivares Tupy, Seleção 97 e Cherokee.

O suco foi elaborado por método caseiro utilizando-se uma mini-suqueira (Ricefer) modelo para $18 \mathrm{~kg}$ como descrito por RIZZON, MANFROI e MENEGUZZO [21]. Neste modelo, o suco é extraído por vapor e o aquecimento é feito por meio de fogareiro a gás. O conjunto é composto por uma cuba para água, coletor de suco e depósito para fruta, inteiramente em aço inox.

Os frutos congelados foram colocados diretamente no extrator e submetidos à ação do vapor de água. $\mathrm{O}$ suco obtido foi pasteurizado $\left(88-90^{\circ} \mathrm{C}\right.$ por $\left.20 \mathrm{~min}\right)$ e engarrafado a quente em garrafas de vidro com capacidade para $215 \mathrm{~mL}$, lacrados com tampa metálica. Não foram empregados aditivos. O suco engarrafado foi armazenado em adega (pouca luminosidade, umidade relativa em torno de $70 \%$ e temperatura entre 16 e $18^{\circ} \mathrm{C}$ ) ou sob refrigeração (refrigerador doméstico à temperatura média de $8^{\circ} \mathrm{C}$ ) para avaliação das alterações decorrentes do armazenamento por até 120 dias. A cada 30 dias foram realizadas análises de pH, SST, acidez (ATT), extrato seco [11], carboidratos [5] e antocianinas [15, 18, 19] nos sucos armazenados nas duas condições de temperatura.

O produto elaborado foi avaliado quanto às suas características físico-químicas como descrito anteriormente para a polpa e quanto à aceitabilidade. $O$ teste de aceitabilidade foi realizado com 16 provadores não treinados. O suco foi diluído na proporção de 50\% (suco:água) e acrescido de açúcar conforme a preferência do provador. Foi ofereci- 
da apenas uma amostra para cada provador que julgou o suco com base em uma escala hedônica de 9 pontos com os extremos gostei muito (9) e desgostei muito (1).

Todas as determinações físico-químicas foram efetuadas em triplicata e os resultados submetidos à análise estatística pelo Programa ESTAT [6]. As análises que apresentaram diferença significativa no teste de variância foram submetidas ao teste de Tukey com nível de significância de 5\%.

\section{3 - RESULTADOS E DISCUSSÃO}

Os dados da Tabela 1 indicam que as cultivares diferem de forma significativa na maior parte dos parâmetros avaliados.

O teor de antocianinas determinado na polpa dos frutos (Tabela 1) confirmou os resultados obtidos no perfil espectral (Figura 1), indicando a possibilidade de separar as cultivares em grupos conforme o teor de antocianinas totais.

O rendimento em suco foi de aproximadamente $85 \%$. Os frutos foram submetidos ao vapor por até $3 \mathrm{~h}$. O rendimento do grupo A foi de $87,6 \%$ após 3 h de extração, seguido do grupo C (84,4\% após 2 h de extração) e grupo B (58\% após extração por $1 \mathrm{~h})$.

As análises físico-químicas dos sucos foram realizadas após resfriamento à temperatura ambiente (Tabela 2).

Os dados obtidos para a polpa e suco (Tabelas 1 e 2) indicam redução nos teores de sólidos solúveis, acidez e carboidratos, provavelmente, pelo acréscimo de água devido à condensação do vapor durante a elaboração do suco. Entretanto, extrações mais rápidas, como a realizada para o grupo B (apenas $1 \mathrm{~h}$ ) não permitiu a extração completa do suco, prejudicando a qualidade do produto final e o rendimento.

GRANADA, VENDRUSCOLO e TREPTOW [9] obtiveram suco de amora-preta por extração com prensa hidráulica com teor mais elevado de sólidos solúveis, em média $7,33^{\circ}$ Brix no tratamento controle, para as cultivares Brazos, Guarani e Tupy. A diferença observada no teor de sólidos solúveis deve ser decorrente da composição da matéria-prima, uma vez que o teor de sólidos solúveis obtido para a polpa da cultivar Tupy neste trabalho foi de $6,93^{\circ} \mathrm{Brix}$, inferior ao observado por estes autores para o suco extraído por prensagem. Outro fator que contribui para a diferença no teor de SST é o método empregado, pois na prensagem não há diluição devido à ação do vapor. A prensagem é um método mais eficiente para a manutenção da composição da fruta, mas exige equipamentos mais sofisticados e tratamento enzimático para melhoria do rendimento e da extração dos compostos que contribuem para as características químicas do suco. Os valores de acidez total obtidos estão dentro da faixa de 0,6 a $1,1 \%$, proposta por SAPERS et al. (1985) e citados por GRANADA, VENDRUSCOLO e TREPTOW [9].

A relação SST/ATT foi de 4,2 para o grupo A e de 5,2 para os grupos B e C. GRANADA, VENDRUSCOLO e TREPTOW [9] obtiveram ratio de 4,74 para suco da cultivar Brazos e 7,42 para a cultivar Tupy, alcançados por prensagem a frio

TABELA 1 - Características físico-químicas da polpa extraída dos frutos de seis cultivares de amora-preta, cultivados na Estação Experimental de Caldas da Epamig (safra 2003/2004)

\begin{tabular}{|c|c|c|c|c|c|c|}
\hline Cultivar & $\mathrm{pH}$ & $\begin{array}{c}\text { SST } \\
\left({ }^{\circ} \text { Brix }\right)\end{array}$ & $\begin{array}{c}\text { Acidez } \\
\text { (\% ácido cítrico) }\end{array}$ & $\begin{array}{c}\text { Carboidratos } \\
(\%)\end{array}$ & $\begin{array}{l}\text { Antocianinas } \\
(\mathrm{mg} / 100 \mathrm{~g})\end{array}$ & $\begin{array}{c}\text { Umidade } \\
(\%)\end{array}$ \\
\hline Guarani & $3,300 \pm 0,006 D^{*}$ & $9,23 \pm 0,15 \mathrm{~B}$ & $1,47 \pm 0,03 \mathrm{~B}$ & $5,92 \pm 0,16 \mathrm{~A}$ & $194,59 \pm 4,72 \mathrm{D}$ & $90,47 \pm 0,26 \mathrm{~B}$ \\
\hline Comanche & $3,266 \pm 0,007 \mathrm{E}$ & $9,15 \pm 0,63$ B & $1,50 \pm 0,02 \mathrm{AB}$ & $4,23 \pm 0,18 \mathrm{C}$ & $121,00 \pm 1,04 \mathrm{AB}$ & $91,77 \pm 0,25 \mathrm{~A}$ \\
\hline Seleção 97 & $3,327 \pm 0,004 \mathrm{C}$ & $9,32 \pm 0,36 \mathrm{AB}$ & $1,26 \pm 0,01 \mathrm{D}$ & $4,90 \pm 0,10 \mathrm{~B}$ & $116,46 \pm 2,50 \mathrm{BC}$ & $91,59 \pm 0,28 \mathrm{~A}$ \\
\hline Cherokee & $3,362 \pm 0,005 B$ & $10,37 \pm 0,47 \mathrm{~A}$ & $1,24 \pm 0,01 \mathrm{D}$ & $5,86 \pm 0,32 \mathrm{~A}$ & $110,21 \pm 0,92 \mathrm{C}$ & $90,74 \pm 0,14 \mathrm{~B}$ \\
\hline
\end{tabular}

*Médias seguidas por letras iguais não diferem entre si pelo teste de Tukey a 5\% de probabilidade

TABELA 2 - Análises físico-químicas dos sucos de amora-preta, separados em três grupos conforme o teor de antocianinas (Grupo A=Guarani; Grupo B=Caingangue, Comanche; Grupo C=Tupy, Seleção 97, Cherokee), logo após o resfriamento

\begin{tabular}{|c|c|c|c|c|c|c|}
\hline Grupo & $\mathrm{pH}$ & $\begin{array}{c}\text { SST } \\
\text { ( }{ }^{\circ} \text { Brix) }\end{array}$ & $\begin{array}{c}\text { Acidez } \\
\text { (\%ácido cítrico) }\end{array}$ & $\begin{array}{c}\text { Carboidratos } \\
(\%)\end{array}$ & $\begin{array}{c}\text { Antocianinas } \\
(\mathrm{mg} / 100 \mathrm{~g})\end{array}$ & $\begin{array}{c}\text { Extrato seco } \\
(\%)\end{array}$ \\
\hline$A$ & $3,28 \pm 0,01 B^{*}$ & $4,87 \pm 0,06$ B & $1,15 \pm 0 \mathrm{~A}$ & $3,78 \pm 0,18 \mathrm{AB}$ & $75,91 \pm 0,82 \mathrm{~A}$ & $4,89 \pm 0,12 \mathrm{~B}$ \\
\hline$B$ & $3,37 \pm 0,02 \mathrm{~A}$ & $4,84 \pm 0 \mathrm{~B}$ & $0,92 \pm 0,02 \mathrm{C}$ & $3,43 \pm 0,04 \mathrm{~B}$ & $65,02 \pm 0,62 \mathrm{C}$ & $4,63 \pm 0,09 \mathrm{C}$ \\
\hline C & $3,24 \pm 0,01 \mathrm{C}$ & $5,34 \pm 0 \mathrm{~A}$ & $1,03 \pm 0,02 \mathrm{~B}$ & $4,05 \pm 0,174 \mathrm{~A}$ & $70,47 \pm 0,47$ B & $5,44 \pm 0,05 \mathrm{~A}$ \\
\hline
\end{tabular}

*Médias seguidas por letras iguais não diferem entre si pelo teste de Tukey a 5\% de probabilidade 
após tratamento com enzimas pectolíticas na polpa. Estes valores indicam que o suco não é adequado para consumo in natura, devendo ser adicionado de açúcar ou associado a sucos de frutas mais doces.

Houve um decréscimo acentuado no teor de antocianinas, sendo observada uma redução maior para o grupo A submetido a $3 \mathrm{~h}$ de extração. O Grupo B, apesar de apresentar níveis superiores de antocianinas em relação ao grupo $\mathrm{C}$, forneceu o suco com o menor índice de antocianinas. Este fato é explicado pelo tempo de extração, pois estes frutos ficaram em contato com o vapor por apenas $1 \mathrm{~h}$, tempo insuficiente para garantir uma extração adequada (Tabela 2). Desta forma, é preciso ajustar o tempo de extração para garantir um bom rendimento e composição química adequada.

Os teores de antocianinas foram superiores aos obtidos por GRANADA, VENDRUSCOLO e TREPTOW [9]. Neste caso, os autores obtiveram teores médios de antocianinas totais de 32,50 mg/100 g. Como estes autores não determinaram o teor de antocianinas na polpa, não é possível determinar o efeito do processamento utilizado na degradação deste composto.

Os valores de antocianinas totais obtidos neste trabalho aproximam-se dos obtidos por WANG e LIN [23] e HASSIMOTTO et al. [10] para os frutos de amora-preta. WANG e LIN [23] compararam o teor de antocianinas totais em frutos verdes e maduros de amora-preta, framboesa e morango e obtiveram teores de 130 a 170 mg/100 g em frutos maduros de diferentes cultivares de amora-preta. Valores semelhantes foram obtidos por HASSIMOTTO et al. [10], cujos resultados indicaram predominância de cianidina com o maior valor obtido para a cultivar Guarani $(156,61 \mathrm{mg} / 100 \mathrm{~g})$ e o menor para a cultivar Brazos $(91,71 \mathrm{mg} / 100 \mathrm{~g})$.
O suco elaborado a partir dos três grupos foi analisado quanto às características físico-químicas após um período de armazenamento de até 120 dias em adega $\left(16-18^{\circ} \mathrm{C}\right)$ ou sob refrigeração $\left(8^{\circ} \mathrm{C}\right)$ (Tabela 3$)$.

Os dados da Tabela 3 indicam que houve influência da temperatura durante o período de armazenamento nas características de SST, acidez e antocianinas.

No armazenamento sob refrigeração houve maior conservação do teor de antocianinas, em média $84 \%$ do teor inicial para os três grupos, enquanto nos sucos armazenados em adega, a perda de antocianinas foi de $30 \%$, em média, após 120 dias de armazenamento (Tabela 4).

Dos parâmetros avaliados, somente o teor de antocianinas apresentou um padrão de decréscimo durante o período de armazenamento. As diferenças observadas nos demais parâmetros devem ter ocorrido devido a pequenas variações de amostragem, uma vez que o desvio padrão obtido entre as repetições foi bastante baixo.

Num estudo sobre os aspectos quantitativos da degradação de antocianinas em morango, MARKAKIS, LIVINGSTON e FELLERS [16], observaram que na presença de oxigênio, a degradação do pigmento foi 10 vezes maior do que o observado no suco de morango armazenado em atmosfera com nitrogênio. Além disso, a reação hidrolítica responsável pela degradação do pigmento apresentou relação direta com a temperatura de estocagem. Daí a necessidade de utilizar a menor temperatura possível em todas as etapas de processamento, embalagem e armazenamento. Quanto ao uso do calor é necessário para reduzir a carga microbiana do produto, os autores recomendam o uso de tratamento a alta temperatura por curto espaço de tempo como forma de garantir maior retenção do pigmento.

TABELA 3 - Características físico-químicas do suco de amora, separados em três grupos conforme o teor de antocianinas (Grupo A=Guarani; Grupo B=Caingangue, Comanche; Grupo C=Tupy, Seleção 97, Cherokee), após armazenamento por $30,60,90$ e 120 dias em adega $\left(16-18^{\circ} \mathrm{C}\right)$ ou geladeira $\left(8^{\circ} \mathrm{C}\right)$

\begin{tabular}{|c|c|c|c|c|c|c|c|}
\hline Grupo & $\begin{array}{c}\text { Tempo de } \\
\text { armaz. (dias) }\end{array}$ & $\mathrm{pH}$ & $\begin{array}{c}\text { SST } \\
\left({ }^{\circ} \text { Brix }\right)\end{array}$ & $\begin{array}{c}\text { Acidez } \\
(\%)\end{array}$ & $\begin{array}{c}\text { Carboidratos } \\
(\%)\end{array}$ & $\begin{array}{c}\text { Antocianinas } \\
(\mathrm{mg} / 100 \mathrm{~g})\end{array}$ & $\begin{array}{c}\text { Extrato Seco } \\
(\%)\end{array}$ \\
\hline \multirow[t]{5}{*}{ A } & 30 & $3,381^{2} A^{3}$ & $4,703 \mathrm{C}$ & $1,079 \mathrm{~A}$ & $3,467 \mathrm{~A}$ & $67,627 \mathrm{~A}$ & $5,215 \mathrm{~A}$ \\
\hline & 60 & $3,277 \mathrm{D}$ & $4,850 \mathrm{~B}$ & $0,957 \mathrm{C}$ & $3,287 \mathrm{AB}$ & $66,735 \mathrm{~A}$ & $5,090 \mathrm{~A}$ \\
\hline & 90 & $3,335 \mathrm{~B}$ & $4,660 \mathrm{D}$ & $0,980 \mathrm{C}$ & $3,467 \mathrm{~A}$ & 61,593 B & $4,908 \mathrm{~B}$ \\
\hline & 120 & $3,310 \mathrm{C}$ & $4,930 \mathrm{~A}$ & $1,044 \mathrm{~B}$ & $3,131 \mathrm{~B}$ & 62,687 B & $4,935 \mathrm{~B}$ \\
\hline & $(T \times D)^{1}$ & $1,629 \mathrm{~ns}$ & $57,000^{* *}$ & $9,111^{* *}$ & $1,983 \mathrm{~ns}$ & $6,303^{* *}$ & $0,129 \mathrm{~ns}$ \\
\hline \multirow[t]{5}{*}{ B } & 30 & $3,408 \mathrm{BC}$ & $4,617 \mathrm{~B}$ & $0,927 \mathrm{~A}$ & $3,243 \mathrm{AB}$ & $56,402 \mathrm{~A}$ & $4,845 \mathrm{~A}$ \\
\hline & 60 & $3,417 \mathrm{~B}$ & $4,683 \mathrm{AB}$ & $0,834 \mathrm{C}$ & $3,235 \mathrm{AB}$ & $52,633 \mathrm{~B}$ & $4,723 \mathrm{~A}$ \\
\hline & 90 & $3,425 \mathrm{~A}$ & $4,477 \mathrm{C}$ & 0,852 C & $3,372 \mathrm{~A}$ & $49,568 \mathrm{C}$ & $4,717 \mathrm{~A}$ \\
\hline & 120 & $3,403 \mathrm{C}$ & $4,730 \mathrm{~A}$ & $0,881 \mathrm{~B}$ & $3,092 \mathrm{~B}$ & $48,594 \mathrm{C}$ & $4,737 \mathrm{~A}$ \\
\hline & $(T x D)^{1}$ & $4,098^{*}$ & $8,493^{* *}$ & $1,944 \mathrm{~ns}$ & $3,084 \mathrm{~ns}$ & $1,322 \mathrm{~ns}$ & $1,537 \mathrm{~ns}$ \\
\hline \multirow[t]{5}{*}{ C } & 30 & $3,316 \mathrm{~A}$ & $5,170 \mathrm{~B}$ & $1,027 \mathrm{~A}$ & $3,689 \mathrm{BC}$ & $62,408 \mathrm{~A}$ & $5,860 \mathrm{~A}$ \\
\hline & 60 & $3,255 \mathrm{C}$ & $5,317 \mathrm{~A}$ & 0,945 B & $3,905 \mathrm{AB}$ & 59,913 B & $5,540 \mathrm{~B}$ \\
\hline & 90 & 3,292 B & $5,100 \mathrm{C}$ & 0,968 B & $4,002 \mathrm{~A}$ & $56,762 \mathrm{C}$ & $5,493 \mathrm{~B}$ \\
\hline & 120 & 3,285 B & $5,327 \mathrm{~A}$ & $1,009 \mathrm{~A}$ & $3,514 \mathrm{C}$ & $53,520 \mathrm{D}$ & $5,508 \mathrm{~B}$ \\
\hline & $(T x D)^{1}$ & $1,891 \mathrm{~ns}$ & $3,859^{*}$ & $10,833^{* *}$ & 1,389 ns & $18,263^{* *}$ & 0,939 ns \\
\hline
\end{tabular}


TABELA 4 - Efeito da temperatura e tempo de armazenamento nos teores de SST, acidez e antocianinas do suco de amora, separados em três grupos conforme o teor de antocianinas (Grupo A=Guarani; Grupo B=Caingangue, Comanche; Grupo C=Tupy, Seleção 97, Cherokee)

\begin{tabular}{|c|c|c|c|c|c|c|c|}
\hline Grupo & $\begin{array}{c}\text { Tempo de } \\
\text { armaz. (dias) }\end{array}$ & $\begin{array}{c}\text { SST } \\
\left(16-18^{\circ} \mathrm{C}\right)\end{array}$ & $\begin{array}{c}\left({ }^{\circ} \mathrm{Brix}\right) \\
\left(8^{\circ} \mathrm{C}\right)\end{array}$ & $\begin{array}{c}\text { Acidez } \\
\left(16-18^{\circ} \mathrm{C}\right)\end{array}$ & $\begin{array}{c}(\%) \\
\left(8^{\circ} \mathrm{C}\right)\end{array}$ & $\begin{array}{c}\text { Antocianinas } \\
\left(16-18^{\circ} \mathrm{C}\right)\end{array}$ & $\begin{array}{c}(\mathrm{mg} / 100 \mathrm{~g}) \\
\left(8^{\circ} \mathrm{C}\right)\end{array}$ \\
\hline \multirow[t]{5}{*}{ A } & 30 & $4,720 \mathrm{C}^{1}$ & $4,687 \mathrm{C}$ & $1,062 \mathrm{~A}$ & $1,097 \mathrm{~A}$ & $63,587 \mathrm{~A}$ & $71,667 \mathrm{~A}$ \\
\hline & 60 & $4,800 \mathrm{~B}$ & $4,900 \mathrm{~A}$ & $0,945 \mathrm{C}$ & $0,968 \mathrm{C}$ & $63,177 \mathrm{~A}$ & $70,293 \mathrm{~A}$ \\
\hline & 90 & $4,710 \mathrm{C}$ & 4,610 D & 0,992 B & $0,968 \mathrm{C}$ & $59,304 \mathrm{~B}$ & $63,883 \mathrm{C}$ \\
\hline & 120 & $5,030 \mathrm{~A}$ & $4,830 \mathrm{~B}$ & $1,073 \mathrm{~A}$ & $1,015 \mathrm{~B}$ & $58,100 \mathrm{~B}$ & 67,273 B \\
\hline & Média geral & $4,815 \mathrm{~A}^{2}$ & $4,757 \mathrm{~B}$ & $1,018 A$ & $1,012 A$ & $61,042 B$ & $68,279 A$ \\
\hline \multirow[t]{5}{*}{ B } & 30 & $4,680 \mathrm{AB}$ & $4,553 \mathrm{~B}$ & $0,933 \mathrm{~A}$ & $0,922 \mathrm{~A}$ & $52,920 \mathrm{~A}$ & $59,880 \mathrm{~A}$ \\
\hline & 60 & $4,633 \mathrm{~B}$ & $4,733 \mathrm{~A}$ & $0,828 \mathrm{C}$ & $0,840 \mathrm{~B}$ & $48,670 \mathrm{~B}$ & $56,600 \mathrm{~B}$ \\
\hline & 90 & $4,510 \mathrm{C}$ & $4,443 \mathrm{C}$ & $0,863 \mathrm{BC}$ & $0,840 \mathrm{~B}$ & $45,254 \mathrm{C}$ & $53,882 \mathrm{C}$ \\
\hline & 120 & $4,730 \mathrm{~A}$ & $4,730 \mathrm{~A}$ & $0,898 \mathrm{~B}$ & $0,863 \mathrm{~B}$ & $44,349 \mathrm{C}$ & $52,840 \mathrm{C}$ \\
\hline & Média geral & $4,638 A$ & $4,615 A$ & $0,881 A$ & $0,866 A$ & $47,796 B$ & $55,802 \mathrm{~A}$ \\
\hline \multirow[t]{5}{*}{$C$} & 30 & $5,170 \mathrm{~B}$ & $5,170 \mathrm{~B}$ & $1,038 \mathrm{~A}$ & $1,015 \mathrm{~A}$ & $59,903 \mathrm{~A}$ & $64,913 \mathrm{~A}$ \\
\hline & 60 & $5,300 \mathrm{~A}$ & $5,330 \mathrm{~A}$ & 0,945 B & $0,945 \mathrm{~B}$ & 57,427 B & $62,400 \mathrm{~B}$ \\
\hline & 90 & $5,100 \mathrm{~B}$ & $5,100 \mathrm{~B}$ & $1,015 \mathrm{~A}$ & $0,922 \mathrm{~B}$ & $53,835 \mathrm{C}$ & $59,689 \mathrm{C}$ \\
\hline & 120 & $5,273 \mathrm{~A}$ & $5,380 \mathrm{~A}$ & $1,003 \mathrm{~A}$ & $1,015 \mathrm{~A}$ & $48,978 \mathrm{D}$ & $58,061 \mathrm{D}$ \\
\hline & Média geral & $5,211 B$ & $5,246 A$ & $1,000 \mathrm{~A}$ & $0,974 B$ & $55,036 B$ & $61,266 A$ \\
\hline
\end{tabular}

${ }^{1}$ Médias seguidas por letras iguais na mesma coluna de cada grupo não diferem entre si pelo teste de Tukey a $5 \%$ de probabilidade

${ }^{2}$ Média dos resultados obtidos em cada temperatura. A comparação foi feita entre as médias obtidas em adega $\left(16-18^{\circ} \mathrm{C}\right)$ e geladeira $\left(8^{\circ} \mathrm{C}\right)$. As letras iguais para as médias gerais de cada parâmetro avaliado indicam que as médias não diferem entre si pelo teste de Tukey a 5\% de probabilidade

O suco de amora diluído e adoçado foi oferecido a 16 provadores não treinados, obtendo uma nota média de 7,9 na escala hedônica de 9 pontos. Considerando o valor máximo da escala como $100 \%$ de aceitação, o suco de amora obtido em extrator caseiro teve aceitabilidade superior a $85 \%$.

\section{4 - CONCLUSÕES}

O extrator de suco, normalmente utilizado pelos viticultores, pode ser empregado na elaboração do suco de amora-preta. É um processo simples e de custo relativamente baixo, que pode ser realizado na pequena propriedade rural a fim de agregar valor aos frutos produzidos e aumentar sua vida de prateleira.

A ação do vapor por $2 \mathrm{~h}$ proporcionou boa extração de compostos e rendimento de $85 \%$. O suco elaborado apresentou boa aceitabilidade, superior a $85 \%$. Entretanto, devido à baixa relação SST/ATT deve ser consumido com adição de açúcar ou edulcorante.

O teor de antocianinas foi reduzido, em média, $42 \%$ durante a elaboração do suco. O tempo de armazenamento também contribuiu para a redução no teor de antocianinas do suco que foi menor nas amostras mantidas sob refrigeração.

O suco pasteurizado e armazenado sob refrigeração manteve-se adequado ao consumo por pelo menos 120 dias, apresentando alteração apenas no teor de antocianinas.

\section{5 - REFERÊNCIAS BIBLIOGRÁFICAS}

[1] ANTUNES, L.E.C. Amora-preta: nova opção de cultivo no Brasil. Ciência Rural, Santa Maria, v. 32, n. 1, p. 151-158, 2002.
[2] ANTUNES, L.E.C.; DUARTE FILHO, J.; SOUZA, C.M. Conservação pós-colheita de frutos de amoreira-preta. Pesquisa Agropecuária Brasileira, Brasília, v. 38, n. 3, p. 413-419, 2003.

[3] CONNOR, A.M.; LUBY, J.J.; HANCOCK, J.F.; BERKHEIMER, S.; HANSON, E.J. Changes in fruit antioxidant activity among blueberry cultivars during cold-temperature storage. Journal of Agricultural and Food Chemistry, v. 50, n. 4, p. 893-898, 2002.

[4] CORDENUNSI, B.R.; NASCIMENTO, J.R.O.; LAJOLO, F.M. Physico-chemical changes related to quality of five strawberry fruit cultivars during cool-storage. Food Chemistry, v. 83, p. 167-173, 2003.

[5] DUBOIS, M.; GILES, K.A.; HAMILTON, J.K.; REBERS, P.A.; SMITH, F. Colorimetric method for determination of sugars and related substances. Analytical Chemistry, v. 28, p. 350, 1956.

[6] ESTAT - Sistema para análises estatísticas. Pólo computacional/Departamento de Ciências Exatas. Unesp-FCAV - Campus de Jaboticabal. V. 2.0.

[7] FOLHA DE S. PAULO. Suco de amora ajuda a combater doenças do coração, diz estudo. Edição de 25 de março de 2003. In: Toda fruta. Curiosidades Gerais. Disponível em: http://www.todafruta.com.br. Acesso em: 7 jan. 2005.

[8] GLOBO REPÓRTER. Mirtilo, o blueberry brasileiro. Edição de 29 de março de 2004. In: Toda Fruta. O poder de cura das frutas. Disponível em: http://www. todafruta.com.br. Acesso em: 11 jan. 2005.

[9] GRANADA, G.L.; VENDRUSCOLO, J.L.; TREPTOW, R.O. Caracterização química e sensorial de sucos clarificados de amora-preta (Rubus spp. L.) Revista Brasileira de Agrociência, v. 7, n. 2, p. 143-147, 2001.

[10] HASSIMOTTO, N.M.A.; GOMEZ, M.L.P.A.; MOTA, R.V.; CORDENUNSI, B.R.; LAJOLO, F.M. Compostos antioxidantes da amora-preta (Rubus sp.). In: XIX Congresso Brasileiro de Ciência e Tecnologia de 
Alimentos: estratégia para o desenvolvimento. 2004, Recife: Anais (cd-rom) XIX CBCTA, n. 424.

[11] INSTITUTO ADOLFO LUTZ. Normas Analíticas do Instituto Adolfo Lutz. Métodos químicos e físicos para análise de alimentos. V. 1, $3^{\text {a }}$ ed. São Paulo: Inst. Adolfo Lutz, 1985, 533 p.

[12] KUSKOSKI, E.M.; ASUERO, A.G.; GARCÍA-PARILLA, M.C.; TRONCOSO, A.M.; FETT, R. Actividad antioxidante de pigmentos antociánicos. Ciência e Tecnologia de Alimentos, Campinas, v. 24, n. 4, p. 691-693, 2004.

[13] LEES, D.H.; FRANCIS, F.J. Standardization of pigment analyses in cranberries. HortScience, v. 7, n. 1, p. 83-84, 1972.

[14] LIMA, V.L.A.G.; GUERRA, N.B. Antocianinas: atividade antioxidante e biodisponibilidade. Boletim da SBCTA, Campinas, v. 37 (Supl.), p. 121-128, 2003.

[15] LIMA, V.L.A.G.; MELO, E.A.; MACIEL, M.I.S.; LIMA, D.E.S. Avaliação do teor de antocianinas em polpa de acerola congelada proveniente de frutos de 12 diferentes aceroleiras (Malpighia emarginata D.C.). Ciência e Tecnologia de Alimentos, Campinas, v. 23, n. 1, p. 101-103, 2003.

[16] MARKAKIS, P; LIVINGSTON, G.E.; FELLERS, C.R. Quantitative aspects of strawberry pigment degradation. Food Research, v. 22, p. 117-130, 1957.

[17] MORENO-ALVAREZ, M.J.; MATOS, A.V.; LÓPEZ, E.; BELÉN, D. Estabilidad de antocianinas em jugos pasteurizados de mora (Rubus glaucus Benth). ALAN, v. 52, n. 2, Supl. 2, 2002, on-line. Disponível em: http://.../scielo.php?script =sci_arttext\&pid $=$ SOOO406222002000200010\&lng =es\&nrm =is.

[18] PERKINS-VEAZIE, P; CLARK, J.R.; HUBER, D.J.; BALDWIN, E.A. Ripening physiology in 'Navaho' thorn- less blackberries: color, respiration, ethylene production, softening, and compositional changes. Journal of American Society for Horticultural Science, v. 125, n. 3, p. 357-363, 2000.

[19] PERKINS-VEAZIE, P.; COLLINS, J.K. Quality of erecttype blackberry fruit after short intervals of controlled atmosphere storage. Postharvest Biology and Technology, v. 25, p. 235-239, 2002.

[20] PERKINS-VEAZIE, P; COLLINS, J.K.; CLARK, J.R. Cultivar and maturity affect postharvest quality of fruit from erect blackberries. HortScience, v. 31, n. 2, p. 258-261, 1996.

[21] RIZZON, L.A.; MANFROI, V.; MENEGUZZO, J. Elaboração de suco de uva na propriedade vitícola. Bento Gonçalves: Embrapa Uva e Vinho, 1998, 24 p. (Embrapa Uva e Vinho. Documentos, 21).

[22] ROMMEL, A.; WROLSTAD, R.E.; HEATHERBELL, D.A. Blackberry juice and wine: processing and storage effects on anthocyanin composition, color and appearance. Journal of Food Science, v. 57, n. 2, p. 385-391, 1992.

[23] WANG, S.Y.; LIN, H.-S. Antioxidant activity in fruits and leaves of blackberry, raspberry, and strawberry varies with cultivar and developmental stage. Journal of Agricultural and Food Chemistry, v. 48, n. 2, p. 140-146, 2000.

\section{6 - AGRADECIMENTOS}

Ao CNPq pela bolsa de recém-doutor. Aos funcionários e pesquisadores da Estação Experimental de Caldas (Epamig/FECD), que não mediram esforços em proporcionar os meios necessários para a realização deste trabalho. 